PROCEEDINGS OF THE

AMERICAN MATHEMATICAL SOCIETY

Volume 137, Number 8, August 2009, Pages 2529-2539

S 0002-9939(09)09872-4

Article electronically published on March 20, 2009

\title{
BLOW-UP FORMULAS AND SMOOTH BIRATIONAL INVARIANTS
}

\author{
ZHAOHU NIE
}

(Communicated by Ted Chinburg)

\begin{abstract}
We prove that the blow-up formula for the singular homology of a complex smooth projective variety with a smooth center respects two natural filtrations, namely the topological and the geometric filtrations. This then enables us to establish some smooth birational invariants.
\end{abstract}

The motivation for this paper is $\mathrm{W}$. Hu's papers $\mathrm{H1}, \mathrm{H} 2, \mathrm{H} 3$, in which he gives some smooth birational invariants of complex smooth projective varieties using Lawson homology and shows that several conjectures involving various filtrations on the singular homology of a complex smooth projective variety are smooth birational statements for certain dimensions. The proofs of these results, after the weak factorization theorem [AKMW, are reduced to the case of one blow up with a smooth center. Then the blow-up formulas for the various filtrations and knowledge in the known cases (top dimension, codimension 1 and dimension 0) would yield most of W. Hu's results in H1, H2, H3 easily and conceptually. This viewpoint is only implicit in the above papers. It is the purpose of this paper to fully establish these blow-up formulas for the various filtrations (see Theorem 1.20). As an application, we define some new smooth birational invariants (see Theorems 1.23 and 1.24).

\section{BACKGROUND AND LIST OF MAIN RESUltS}

We work over $\mathbb{C}$. Let $X$ be a projective variety of dimension $n$, which in all our results is assumed to be smooth. We write $H_{k}(X)$ for integral singular homology. We now introduce various filtrations on $H_{k}(X)$.

For the topological filtration, first define the Lawson homology of $X$ to be L1

$$
L_{p} H_{k}(X)= \begin{cases}\pi_{k-2 p} \mathcal{Z}_{p}(X), & k \geq 2 p \geq 0 \\ H_{k}(X), & k \geq 2 p, \quad p<0 \\ 0, & k<2 p\end{cases}
$$

where $\mathcal{Z}_{p}(X)$ is the group of algebraic $p$-cycles (integral combinations of $p$-dimensional irreducible subvarieties) on $X$ with a suitable topology (induced from the Chow variety construction).

Received by the editors October 1, 2007, and, in revised form, September 30, 2008.

2000 Mathematics Subject Classification. Primary 14F43, 14 E99.

Key words and phrases. Lawson homology, topological filtration, geometric filtration, blow-up formula, birational invariants.

(C)2009 American Mathematical Society Reverts to public domain 28 years from publication 
Note that by the Dold-Thom theorem [DT,

$$
L_{0} H_{k}(X)=\pi_{k} \mathcal{Z}_{0}(X)=H_{k}(X) .
$$

Lawson's complex suspension theorem [L1 asserts that

$$
\Sigma: \mathcal{Z}_{p}(X) \stackrel{\sim}{\rightarrow} \mathcal{Z}_{p+1}(\Sigma X), p \geq 0,
$$

is a homotopy equivalence, where $\Sigma$ is the operation of complex suspension (producing, for any projective variety, the Thom space of the line bundle $\mathcal{O}(1)$ on it, which is just the projective cone and hence a projective variety).

There is a natural transformation, called the cycle class map [FM],

$$
\sigma: L_{p} H_{k}(X) \rightarrow H_{k}(X), p \geq 0,
$$

which is based on (1.2) and iteration of the s-map (loc. cit.)

$$
s: \mathcal{Z}_{p}(X) \rightarrow \Omega^{2} \mathcal{Z}_{p-1}(X), p \geq 1,
$$

which in turn is based on (1.3) and defined up to homotopy.

Lima-Filho $[\mathrm{LF}$ extends the above definition (1.1) to a quasi-projective variety $U$. For $p \geq 0$, one defines

$$
\mathcal{Z}_{p}(U)=\mathcal{Z}_{p}(X) / \mathcal{Z}_{p}\left(X_{\infty}\right)
$$

as a topological abelian group, where $X$ is a compactification of $U$ with complement $X_{\infty}$. The topology of $\mathcal{Z}_{p}(U)$ is independent of the choice of $X$. For $p<0$, one replaces the singular homology in (1.1) by the Borel-Moore homology $H_{k}^{\mathrm{BM}}(U)$. Again when $p=0$, by the relative version of the Dold-Thom theorem [DT, one has

$$
L_{0} H_{k}(U)=H_{k}\left(X, X_{\infty}\right)=H_{k}^{\mathrm{BM}}(U) .
$$

Friedlander and Gabber [FG] reformulate the complex suspension theorem (1.3) by asserting that the pullback map

$$
\text { p.b. : } \mathcal{Z}_{p}(U) \stackrel{\sim}{\rightarrow} \mathcal{Z}_{p+t}(F), p \geq 0
$$

is a homotopy equivalence for a complex vector bundle $F \rightarrow U$ of rank $t$.

Remark 1.9. The current definition for $L_{p} H_{k}(X)$ when $p<0$ in (1.1) is a "reduced" one, whose stabilization uses (1.8) instead of an iteration of (1.3). This reduction is the key to making everything work in (1.15) and (1.21).

Definition 1.10 ([FM]). The topological filtration $\left\{T_{p} H_{k}(X)\right\}$ on $H_{k}(X)$ is defined by

$$
T_{p} H_{k}(X):=\operatorname{Im}\left(\sigma: L_{p} H_{k}(X) \rightarrow H_{k}(X)\right) .
$$

The geometric filtration $\left\{G_{p} H_{k}(X)\right\}$ on $H_{k}(X)$ is defined by

$$
G_{p} H_{k}(X):=\sum_{\operatorname{dim} Y \leq k-p} \operatorname{Im}\left(H_{k}(Y) \rightarrow H_{k}(X)\right),
$$

where the summation runs over subvarieties of dimension $\leq k-p$. (The geometric filtration $\left\{G_{p} H_{k}(X)\right\}$ is often referred to as the niveau filtration and denoted by $\left\{N_{p} H_{k}(X)\right\}$.)

Both of the above two filtrations are decreasing ones. (For $T_{p} H_{k}$, one notes that (1.4) is defined iteratively by the $s$ map (1.5).) It is clear that

$$
T_{p} H_{k}(X)=G_{p} H_{k}(X)=H_{k}(X), p \leq 0,
$$

by (1.1), (1.2) and the weak Lefschetz theorem. 
Remark 1.12. There are various conjectures to relate the above filtrations, and also with Grothendieck's corrected Hodge filtration for rational coefficients. See [FM].

Now let's list the main results of this paper, whose proofs will be given in subsequent sections.

Let $E \rightarrow X$ be a complex vector bundle of rank $s+1$, and $\varpi: \mathbb{P}(E) \rightarrow X$ its projectivization. One has the following well-known projective bundle formula for singular homology (see e.g. [Le, Theorem 8.23] for the cohomological version):

$$
\psi_{s}=\sum_{i=0}^{s} \psi_{s}^{i}=\sum_{i=0}^{s} c_{1}(\mathcal{O}(1))^{s-i} \circ \varpi^{*}: \bigoplus_{i=0}^{s} H_{k-2 i}(X) \stackrel{\sim}{\rightarrow} H_{k}(\mathbb{P}(E)),
$$

where $\varpi^{*}: H_{*}(X) \rightarrow H_{*+2 s}(\mathbb{P}(E))$ is the pullback map, and $c_{1}(\mathcal{O}(1)): H_{*}(\mathbb{P}(E)) \rightarrow$ $H_{*-2}(\mathbb{P}(E))$ is the cap product with the first Chern class of the canonical line bundle $\mathcal{O}(1)$ on $\mathbb{P}(E)$, which is the dual of the tautological subbundle of $\varpi^{*} E$.

Theorem 1.14. The formula (1.13) respects both filtrations; i.e. one has the following projective bundle formulas:

$$
\begin{aligned}
\psi_{T} & : \bigoplus_{i=0}^{s} T_{p-i} H_{k-2 i}(X) \stackrel{\sim}{\rightarrow} T_{p} H_{k}(\mathbb{P}(E)), \\
\psi_{G}: & \bigoplus_{i=0}^{s} G_{p-i} H_{k-2 i}(X) \stackrel{\stackrel{\sim}{\rightarrow}}{\rightarrow} G_{p} H_{k}(\mathbb{P}(E)),
\end{aligned}
$$

where the maps are the restrictions of $\psi_{s}$ in (1.13).

Lawson homology has a projective bundle formula [FG, [H1]. To prove (1.15), we study the natural transformation from the Lawson formula to the singular formula (see Proposition 2.5).

Using the above projective bundle formulas as one ingredient, we can prove the following blow-up formulas, which is the main tool for our applications.

Let $Y \subset X$ be a smooth subvariety of codimension $r \geq 2$, and $\eta: \widetilde{X_{Y}} \rightarrow X$ the blow up of $X$ with center $Y$. Note the following fiber square:

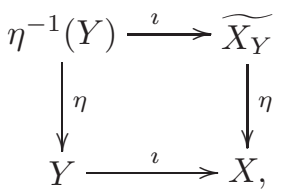

where the exceptional divisor

$$
\eta^{-1}(Y)=\mathbb{P}(N)
$$

is the projectivization of the normal bundle $N$ of $Y$ in $X$. It is well known that one has the following blow-up formula for singular homology (see e.g. Le, Proposition 13.1] for the cohomological version):

$$
I_{s}=\eta^{*}+\sum_{i=1}^{r-1} \imath_{*} \psi_{s}^{i}: H_{k}(X) \oplus \bigoplus_{i=1}^{r-1} H_{k-2 i}(Y) \stackrel{\sim}{\longrightarrow} H_{k}\left(\widetilde{X_{Y}}\right),
$$

where $\eta^{*}: H_{*}(X) \rightarrow H_{*}\left(\widetilde{X_{Y}}\right)$ is the Gysin map, $\imath_{*}: H_{*}\left(\eta^{-1}(Y)\right) \rightarrow H_{*}\left(\widetilde{X_{Y}}\right)$ is induced by the inclusion, and the $\psi_{s}^{i}$ are as in (1.13) in view of (1.18). 
Theorem 1.20. The formula (1.19) respects both filtrations; i.e. one has the following blow-up formulas:

$$
\begin{gathered}
I_{T}: T_{p} H_{k}(X) \oplus \bigoplus_{i=1}^{r-1} T_{p-i} H_{k-2 i}(Y) \stackrel{\sim}{\rightarrow} T_{p} H_{k}\left(\widetilde{X_{Y}}\right), \\
I_{G}: G_{p} H_{k}(X) \oplus \bigoplus_{i=1}^{r-1} G_{p-i} H_{k-2 i}(Y) \stackrel{\sim}{\rightarrow} G_{p} H_{k}\left(\widetilde{X_{Y}}\right),
\end{gathered}
$$

where the maps are the restrictions of $I_{s}$ in (1.19).

Lawson homology also has a blow-up formula [H1]. For (1.21), we study the natural transformation from the Lawson formula to the singular formula (see Proposition 3.8 .

Here is an application of Theorem [1.20, employing the weak factorization theorem AKMW which asserts that any birational map between smooth projective varieties can be factorized as a composition of either blowing up or blowing down with smooth centers.

Theorem 1.23. Let $X$ be a smooth projective variety over $\mathbb{C}$. The first graded parts of the topological filtration and the geometric filtration,

$$
\begin{aligned}
G r_{1}^{T} H_{k}(X) & =H_{k}(X) / T_{1} H_{k}(X), \\
G r_{1}^{G} H_{k}(X) & =H_{k}(X) / G_{1} H_{k}(X),
\end{aligned}
$$

are smooth birational invariants.

In the same spirit, we also have the following result.

Theorem 1.24. Let $X$ be a smooth projective variety over $\mathbb{C}$. Both the kernel and the cokernel of the following weight 2 regulator maps

$$
R: H_{\mathcal{M}}^{k}(X, \mathbb{Z}(2)) \rightarrow H_{\mathcal{D}}^{k}(X, \mathbb{Z}(2)), \forall k \leq 4,
$$

from motivic cohomology to Deligne cohomology, are smooth birational invariants.

\section{Projective Bundle formulas}

In this section, we prove Theorem 1.14.

We first consider the topological filtration and therefore Lawson homology. The projective bundle formula for Lawson homology was first proved by Friedlander and Gabber [FG] and then generalized by W. Hu [H1].

Theorem 2.1 ([FG]). One has the following natural homotopy equivalence:

$$
\phi_{L}=\sum_{i=0}^{s} \phi_{L}^{i}:=\sum_{i=0}^{s} c_{1}(\mathcal{O}(1))^{s-i} \circ \varpi^{*}: \bigoplus_{i=0}^{s} \mathcal{Z}_{q+s-i}(X) \stackrel{\sim}{\rightarrow} \mathcal{Z}_{q+s}(\mathbb{P}(E)),
$$

where $q \geq 0, \varpi^{*}: \mathcal{Z}_{*}(X) \rightarrow \mathcal{Z}_{*+s}(\mathbb{P}(E))$ is the flat pullback map, and $c_{1}(\mathcal{O}(1))$ : $\mathcal{Z}_{*}(\mathbb{P}(E)) \rightarrow \mathcal{Z}_{*-1}(\mathbb{P}(E))$ is the intersection with the canonical line bundle $\mathcal{O}(1)$ on $\mathbb{P}(E)$, which is defined in $[\mathrm{FG}$ ] based on (1.8) and up to homotopy. 
As a consequence, upon applying $\pi_{k-2 p}$ one gets a projective bundle formula on the Lawson homology level:

$$
\psi_{L}:=\pi_{k-2 p}\left(\phi_{L}\right)=\sum_{i=0}^{s} \psi_{L}^{i}: \bigoplus_{i=0}^{s} L_{p-i} H_{k-2 i}(X) \stackrel{\sim}{\rightarrow} L_{p} H_{k}(\mathbb{P}(E))
$$

where $p=q+s \geq s$.

Theorem 2.4 ([1] $)$. The above formula (2.3) also holds for any $p \geq 0$.

In the following proposition, we give an explicit construction of formula (2.3) for any $p$ (cf. [H1]), so that we also establish the natural transformation from this formula to the projective bundle formula (1.13) in singular homology via the cycle class map $\sigma$ (1.4).

Proposition 2.5. For any $p \geq 0$, one has the following commutative diagram:

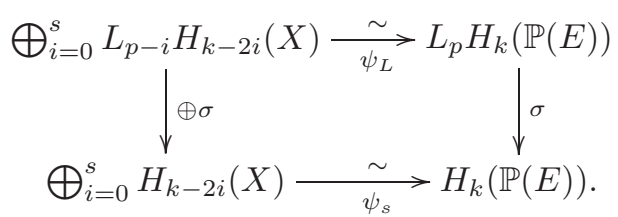

Proof. If $p \geq s$, then we use (2.3) for the top row. Comparing (1.13) and (2.2), one arrives at the commutativity, since the $s$-map (1.5) clearly commutes with flat pullback and intersection with a line bundle.

If $p<s$, we multiply the base $X$ by $\mathbb{C}^{s-p}$. Observe that the pullback of $E$ to $X \times \mathbb{C}^{s-p}$ is $E \times \mathbb{C}^{s-p}$, and hence its projectivization is $\mathbb{P}(E) \times \mathbb{C}^{s-p}$. We now proceed as follows:

$$
\begin{aligned}
& \bigoplus_{i=0}^{p} \mathcal{Z}_{p-i}(X) \oplus \bigoplus_{i=p+1}^{s} \mathcal{Z}_{0}\left(X \times \mathbb{C}^{i-p}\right) \stackrel{\text { p.b., } \sim}{\longrightarrow} \bigoplus_{i=0}^{s} \mathcal{Z}_{s-i}\left(X \times \mathbb{C}^{s-p}\right) \\
& \stackrel{\phi_{L}, \sim}{\longrightarrow} \mathcal{Z}_{s}\left(\mathbb{P}(E) \times \mathbb{C}^{s-p}\right) \stackrel{\text { p.b., } \sim}{\longleftarrow} \mathcal{Z}_{p}(\mathbb{P}(E)),
\end{aligned}
$$

where the pullbacks "p.b." are homotopy equivalences by (1.8), and the second homotopy equivalence is by (2.2). We invert the last homotopy equivalence and consider the composition.

Now applying $\pi_{k-2 p}$ to the above map, we get the isomorphism (2.3) for $p<s$, as long as we note the following: When $p+1 \leq i \leq s$,

$$
\pi_{k-2 p} \mathcal{Z}_{0}\left(X \times \mathbb{C}^{i-p}\right) \stackrel{1.7}{=} H_{k-2 p}^{\mathrm{BM}}\left(X \times \mathbb{C}^{i-p}\right) \cong H_{k-2 i}(X)=L_{p-i} H_{k-2 i}(X),
$$

where the second step is basically the suspension isomorphism for singular homology and the last step is by definition (1.1). 
Using this definition of $\psi_{L}$, one has the following commutative diagram when $p<s$ :

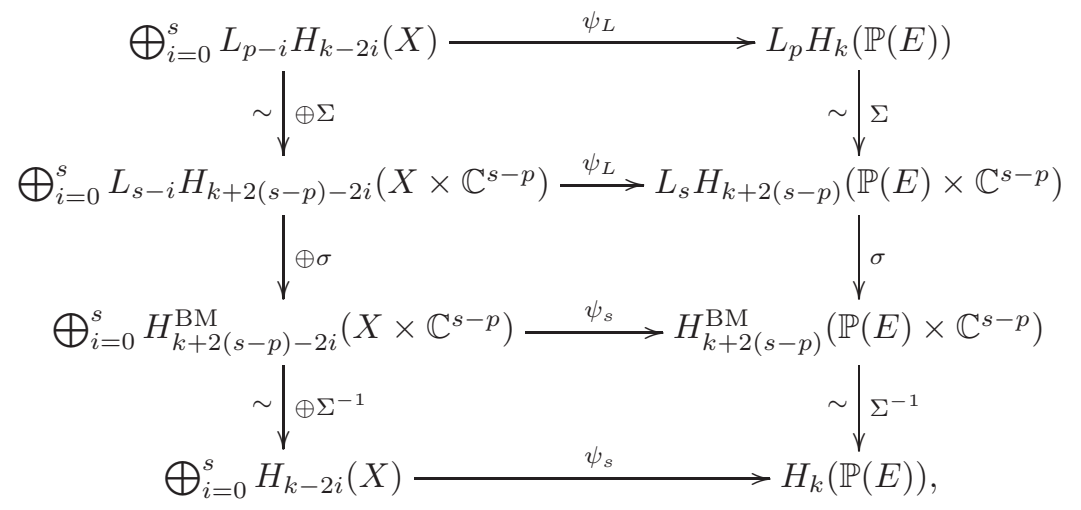

where the $\Sigma$ 's are complex (cf. (1.8) ) and singular suspension isomorphisms, and the $\sigma$ 's are the cycle class maps. Note that the compositions of both columns are just the cycle class maps in (2.6), since the cycle class maps intervene with the complex and singular suspension isomorphism (see [FM, Corollary on pg. 63]). Since all three squares are commutative by our above definition, the $p \geq s$ case and clear reason, one arrives at the commutativity of (2.6) for $p<s$.

Now let's prove Theorem 1.14

Proof of Theorem 1.14. (1.15) follows from the above Proposition 2.5. We now study (1.16).

First we observe that $\psi_{s}$ (1.13) maps the LHS to the RHS of (1.16): If $z_{i} \in$ $G_{p-i} H_{k-2 i}(X)$ is supported on a subvariety $Z_{i}$ of dimension $\leq k-p-i$ (i.e. $z_{i}$ is in the image of $\left.H_{k-2 i}\left(Z_{i}\right) \rightarrow H_{k-2 i}(X)\right)$, then $\varpi^{*}\left(z_{i}\right)$ is supported on $\varpi^{*}\left(Z_{i}\right)$, which has dimension $\leq k-p+s-i$, and $c_{1}(\mathcal{O}(1))^{s-i} \circ \varpi^{*}\left(z_{i}\right)$ is supported on the $(s-i)$-th intersection of $\varpi^{*}\left(Z_{i}\right)$ with $\mathcal{O}(1)$. By Chow's moving lemma Ro, we can move the zero locus of a section of $\mathcal{O}(1)$ in its rational equivalence class to make the intersection proper. The intersection has dimension $\leq k-p$ and supports $\psi_{s}\left(z_{i}\right)$. Hence $\psi_{s}\left(z_{i}\right) \in G_{p} H_{k}(\mathbb{P}(E))$.

Therefore the restriction $\psi_{G}$ is well defined, and it is clearly injective, being the restriction of the isomorphism $\psi_{s}$. Now let's prove that it is surjective.

Suppose that $z \in G_{p} H_{k}(\mathbb{P}(E))$ is supported on a subvariety $Z \subset \mathbb{P}(E)$ of dimension $\leq k-p$. By (1.13), one has a canonical decomposition

$$
z=\sum_{i=0}^{s} \psi_{s}^{i}\left(z_{i}\right)=\sum_{i=0}^{s} c_{1}(\mathcal{O}(1))^{s-i} \circ \varpi^{*} z_{i}
$$

with $z_{i} \in H_{k-2 i}(X)$. We now show that

$$
z_{i} \in G_{p-i} H_{k-2 i}(X)
$$

for $0 \leq i \leq s$ using induction on $i$.

First note that

$$
\varpi_{*}\left(c_{1}(\mathcal{O}(1))^{s-j} \circ \varpi^{*} \alpha\right)= \begin{cases}0, & \text { if } j>0 \\ \alpha, & \text { if } j=0\end{cases}
$$

for any $\alpha \in H_{*}(X)$. 
For $i=0$, it is clear that $Z_{0}:=\varpi_{*}(Z)$ has dimension $\leq k-p$ and supports $z_{0}=\varpi_{*}(z)$ by (2.7) and (2.8).

Now assume that $z_{j}$ is supported on a subvariety $Z_{j}$ of dimension $\leq k-p-j$ for $0 \leq j \leq i-1$. Applying $\varpi_{*}\left(c_{1}(\mathcal{O}(1))^{i} \circ-\right)$ to (2.7) and in view of (2.8), one has

$$
z_{i}=\varpi_{*}\left(c_{1}(\mathcal{O}(1))^{i} \circ z\right)-\sum_{j=0}^{i-1} \varpi_{*}\left(c_{1}(\mathcal{O}(1))^{s-j+i} \circ \varpi^{*} z_{j}\right) .
$$

It is now clear that $z_{i}$ is supported on the support $Z_{i}$ of the following cycle:

$$
\varpi_{*}\left(c_{1}(\mathcal{O}(1))^{i} \circ Z\right)-\sum_{j=0}^{i-1} \varpi_{*}\left(c_{1}(\mathcal{O}(1))^{s-j+i} \circ \varpi^{*} Z_{j}\right),
$$

where the intersection $c_{1}(\mathcal{O}(1)) \circ$ is well defined up to rational equivalence using Chow's moving lemma to move the zero locus of a section of $\mathcal{O}(1)$. Clearly $Z_{i}$ has dimension $\leq k-p-i$ by the induction hypothesis. We are done.

\section{BLOW-UP FORMULAS}

In this section, we prove Theorem 1.20 ,

Again we first consider the topological filtration and hence Lawson homology. The blow-up formula for Lawson homology is proved in [H1].

Theorem 3.1 ([H1]). With the notation as above, one has a natural isomorphism

$$
I_{L}=\eta^{*}+\sum_{i=1}^{r-1} \imath_{*} \psi_{L}^{i}: L_{p} H_{k}(X) \oplus \bigoplus_{i=1}^{r-1} L_{p-i} H_{k-2 i}(Y) \stackrel{\sim}{\rightarrow} L_{p} H_{k}\left(\widetilde{X_{Y}}\right),
$$

where the Gysin map $\eta^{*}$ is defined by $\mathrm{FG}, \mathrm{Pe}$, the $\psi_{L}^{i}$ are as in (2.3) in view of (1.18), and $\imath_{*}$ is induced by the closed embedding in (1.17).

Like the version of the projective bundle formula in Theorem 2.1] by [FG], one can first formulate the above result in terms of algebraic cycles.

Proposition 3.2. With the notation as above, one has an exact sequence of topological abelian groups

$$
0 \rightarrow \mathcal{Z}_{p}\left(\eta^{-1}(Y)\right) \stackrel{\eta_{*} \oplus \imath_{*}}{\longrightarrow} \mathcal{Z}_{p}(Y) \oplus \mathcal{Z}_{p}\left(\widetilde{X_{Y}}\right) \stackrel{-\imath_{*}+\eta_{*}}{\longrightarrow} \mathcal{Z}_{p}(X) \rightarrow 0
$$

After one inverts homotopy equivalences in the category of topological abelian groups, there is a map

$$
\eta^{*}: \mathcal{Z}_{p}(X) \rightarrow \mathcal{Z}_{p}\left(\widetilde{X_{Y}}\right)
$$

which splits (3.3).

Therefore one gets a homotopy equivalence

$$
\left(\begin{array}{cc}
\eta_{*} & 0 \\
\imath_{*} & \eta^{*}
\end{array}\right): \mathcal{Z}_{p}\left(\eta^{-1}(Y)\right) \oplus \mathcal{Z}_{p}(X) \stackrel{\sim}{\rightarrow} \mathcal{Z}_{p}(Y) \oplus \mathcal{Z}_{p}\left(\widetilde{X_{Y}}\right) .
$$

When $p \geq r-1$, one has the following homotopy equivalence:

$$
J:=\eta^{*}+\sum_{i=1}^{r-1} \imath_{*} \phi_{L}^{i}: \mathcal{Z}_{p}(X) \oplus \bigoplus_{i=1}^{r-1} \mathcal{Z}_{p-i}(Y) \stackrel{\sim}{\rightarrow} \mathcal{Z}_{p}\left(\widetilde{X_{Y}}\right)
$$

where the $\phi_{L}^{i}$ are as in (2.2) in view of (1.18). 
Proof. Let $U=\widetilde{X_{Y}}-\eta^{-1}(Y)=X-Y$. Note the following diagram of exact sequences of abelian groups:

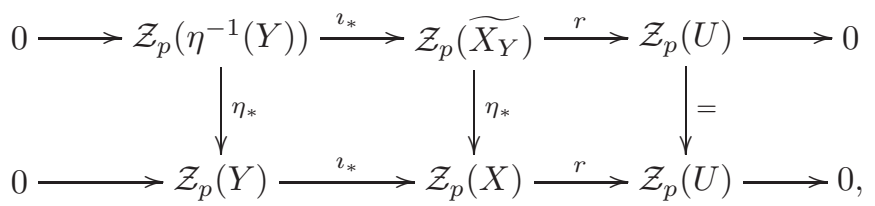

where we use (1.6) for the topology on $\mathcal{Z}_{p}(U)$, and the $r$ 's are the natural restriction (or projection) maps.

Now the exactness of (3.3) follows from (3.7) by a direct diagram-chasing.

Up to homotopy, the map $\eta^{*}$ in (3.4) is defined by [FG. It is a splitting since $\eta_{*} \circ \eta^{*}=i d$ up to homotopy by [Pe, Lemma $\left.11 \mathrm{c}\right]$.

Then (3.5) follows easily from (3.3) and (3.4).

Now (3.6) follows from (3.5) in view of (1.18) and Theorem 2.1. as long as one notes that the composition

$$
\mathcal{Z}_{p}(Y) \stackrel{\phi_{L}^{0}}{\longrightarrow} \mathcal{Z}_{p}\left(\eta^{-1}(Y)\right) \stackrel{\eta_{*}}{\rightarrow} \mathcal{Z}_{p}(Y)
$$

is the identity up to homotopy (cf. the $j=0$ case in (2.8) ).

Now upon applying $\pi_{k-2 p}$ to (3.6), one gets Theorem 3.1 when $p \geq r-1$. If $p<r-1$, then we can apply the same technique as in the proof of Proposition 2.5. Multiply everything by $\mathbb{C}^{k}$ for $k=r-1-p$ and apply Lawson's complex suspension

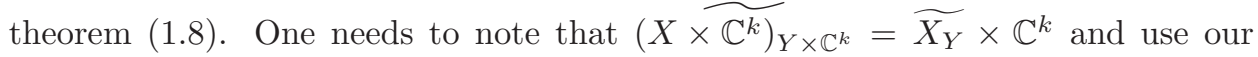
convention (1.1) for $L_{<0} H_{*}$.

Proposition 3.8. With the notation as above, one has the following commutative diagram of blow-up formulas:

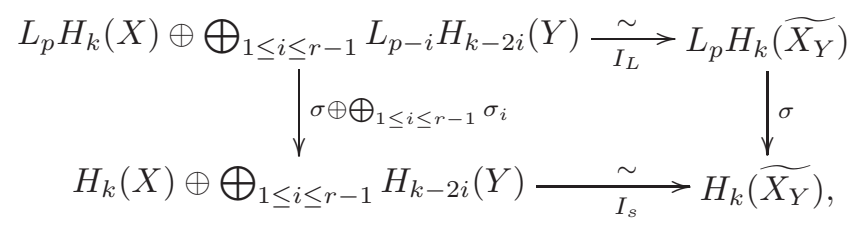

where the $\sigma$ 's are the cycle class maps (1.4).

Proof. When $p \geq r-1$, one compares the explicit formula (3.6) to the singular homology case (1.19) and notes that the $s$-map (1.5) commutes with the Gysin map $\eta^{*}$ (cf. (3.9) ). Otherwise, one uses a procedure similar to that in the proof of Proposition 2.5.

Now let's prove Theorem 1.20

Proof of Theorem 1.20, Again, the topological formula (1.21) follows from Proposition 3.8 We concentrate on the geometric formula (1.22).

First of all, we note that $I_{s}$ in (1.19) induces a map on the geometric filtration level: The Gysin map is defined by

$$
\eta^{*}: H_{k}(X) \rightarrow H_{k}\left(\widetilde{X_{Y}}\right) ; z \mapsto p r_{1 *}\left(\Gamma_{\eta} \circ p r_{2}^{*}(z)\right),
$$

where $p r_{1}: \widetilde{X_{Y}} \times X \rightarrow \widetilde{X_{Y}}$ and $p r_{2}: \widetilde{X_{Y}} \times X \rightarrow X$ are the projections, $\Gamma_{\eta} \subset \widetilde{X_{Y}} \times X$ is the graph of $\eta$, which also denotes its homology class in $H_{n}\left(\widetilde{X_{Y}} \times X\right)$ by abusing 
notation, and $\circ$ denotes the intersection product. It is clear that flat pullback, intersection with the fundamental class of a smooth subvariety and projection are all compatible with the geometric filtration. Therefore so is $\eta^{*}$.

Being the restriction of an isomorphism, (1.22) is clearly an injection, and now we want to prove that it is a surjection.

Suppose that $z \in G_{p} H_{k}\left(\widetilde{X_{Y}}\right)$ is supported on a subvariety $Z \subset \widetilde{X_{Y}}$ of dimension $\leq k-p$. By (1.19), we can write

$$
z=I_{s}\left(z_{0}, z_{1}, \ldots, z_{r-1}\right)
$$

where $z_{0} \in H_{k}(X)$, and $z_{i} \in H_{k-2 i}(Y)$ for $1 \leq i \leq r-1$. We now produce cycles $Z_{0} \subset X$ of dimension $\leq k-p$ such that $z_{0}$ is supported on $Z_{0}$, and $Z_{i} \subset Y$ of dimension $\leq k-p-i$ such that $z_{i}$ is supported on $Z_{i}$ for $1 \leq i \leq r-1$. We take $Z_{0}=\eta_{*}(Z)$. Then $z_{0}$ is supported on $Z_{0}$ since $z_{0}=\eta_{*}(z)$. To produce the $Z_{i}$ for $1 \leq i \leq r-1$, we now produce $W \subset \eta^{-1}(Y)$ of dimension $\leq k-p$, which supports $w=z-\eta^{*} z_{0}=z-\eta^{*} \eta_{*} z$, and then apply the projective bundle formula (1.16). Actually, we take $W$ to be the support of the cycle $Z-\eta^{*} \eta_{*} Z$, where $\eta^{*}$ is the proper transform. It is clear that $W$ is in $\eta^{-1}(Y)$, and it supports $w$.

Remark 3.10. Arapura and Kang AK1, AK2 have many results related to (the dual cohomological version of) (1.22).

\section{Smooth BIRATIONAL InVARIANTS}

We now prove our new smooth birational invariants in Theorems 1.23 and 1.24

Proof of Theorem 1.23. In view of the blow-up formulas (1.19), (1.21) and (1.22) for $p=1$, to show that

$$
\begin{gathered}
H_{k}(X) / T_{1} H_{k}(X) \stackrel{\sim}{\rightarrow} H_{k}\left(\widetilde{X_{Y}}\right) / T_{1} H_{k}\left(\widetilde{X_{Y}}\right) \text { and } \\
H_{k}(X) / G_{1} H_{k}(X) \stackrel{\sim}{\rightarrow} H_{k}\left(\widetilde{X_{Y}}\right) / G_{1} H_{k}\left(\widetilde{X_{Y}}\right),
\end{gathered}
$$

one only needs to show that

$$
T_{1-i} H_{k-2 i}(Y)=G_{1-i} H_{k-2 i}(Y)=H_{k-2 i}(Y),
$$

for $1 \leq i \leq r-1$. This clearly holds by (1.11).

As a corollary, one has an isomorphism

$$
\sigma: L_{1} H_{k}(X) \stackrel{\sim}{\rightarrow} H_{k}(X), k \geq 2
$$

for a smooth rational projective variety $X$. [H1] proves the injectivity, and the surjectivity follows from Theorem 1.23 and the corresponding result for the projective spaces [L1].

Proof of Theorem 1.24. We refer the reader to [EV] for the definition of Deligne cohomology and to Bl] for a definition of the regulator maps in terms of higher Chow groups, which are isomorphic to motivic cohomology for smooth varieties. Both motivic and Deligne cohomology have blow-up formulas, which the regulator maps respect. Actually both cohomology theories can be defined for Chow motives, 
and the blow-up formula holds in Chow motives (see Ma ). Therefore, using our usual notation, we have the following commutative diagram:

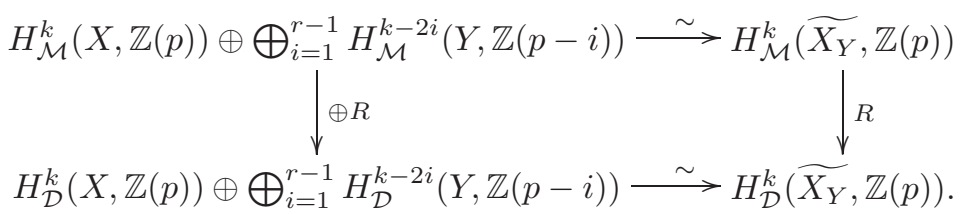

Now consider the case $p=2$ and $k \leq 4$. Then $2-i \leq 1$ for $1 \leq i \leq r-1$ with $r \geq 2$. Then our theorem follows from the following isomorphisms:

$$
\begin{gathered}
H_{\mathcal{M}}^{j}(Y, \mathbb{Z}(1)) \cong H_{\mathcal{D}}^{j}(Y, \mathbb{Z}(1))= \begin{cases}\operatorname{Pic}(Y), & \text { if } j=2, \\
\mathcal{O}^{*}(Y), & \text { if } j=1, \\
0, & \text { if } j<1 ;\end{cases} \\
H_{\mathcal{M}}^{j}(Y, \mathbb{Z}(0)) \cong H_{\mathcal{D}}^{j}(Y, \mathbb{Z}(0))= \begin{cases}\mathbb{Z}, & \text { if } j=0, \\
0, & \text { if } j<0 ;\end{cases} \\
H_{\mathcal{M}}^{j}(Y, \mathbb{Z}(q)) \cong H_{\mathcal{D}}^{j}(Y, \mathbb{Z}(q))=0, \quad \forall q<0, j .
\end{gathered}
$$

\section{REFERENCES}

[AKMW] Abramovich, D.; Karu, K.; Matsuki, K.; Włodarczyk, J. Torification and factorization of birational maps. J. Amer. Math. Soc. 15 (2002), no. 3, 531-572 (electronic). MR:1896232(2003c:14016)

[AK1] Arapura, D.; Kang, S.-J. Functoriality of the coniveau filtration. Canad. Math. Bull. 50 (2007), no. 2, 161-171. MR2317438(2008f:14018)

[AK2] Arapura, D.; Kang, S.-J. Coniveau and the Grothendieck group of varieties. Michigan Math. J. 54 (2006), no. 3, 611-622. MR2280497 (2007k:14011)

[Bl] Bloch, S. Algebraic cycles and the Bellinson conjectures. The Lefschetz centennial conference, Part I (Mexico City, 1984), 65-79, Contemp. Math., 58, Amer. Math. Soc., Providence, RI, 1986. MR860404 (88e:14006)

[DT] Dold, A.; Thom, R. Quasifaserungen und unendliche symmetrische Produkte (German). Ann. of Math. (2) 67 (1958), 239-281. MR0097062(20:3542)

[EV] Esnault, H. and Viehweg, E. Deligne-Beìlinson cohomology. Beyllinson's conjectures on special values of $L$-functions, 43-91, Perspect. Math., 4, Academic Press, Boston, MA, 1988. MR944991 (89k:14008)

[FG] Friedlander, E. M.; Gabber, O. Cycle spaces and intersection theory. Topological methods in modern mathematics (Stony Brook, NY, 1991), 325-370, Publish or Perish, Houston, TX, 1993. MR1215970 (94j:14010)

[FM] Friedlander, E. M.; Mazur, B. Filtrations on the homology of algebraic varieties. With an appendix by Daniel Quillen. Mem. Amer. Math. Soc. 110 (1994), no. 529, x+110 pp. MR.1211371 (95a:14023)

[H1] Hu, W. Birational invariants defined by Lawson homology. To appear in Int. J. Pure Appl. Math. arXiv:math/0511722.

[H2] Hu, W. The Generalized Hodge conjecture for 1-cycles and codimension two algebraic cycles. arXiv:math/0511725.

[H3] Hu, W. Some relations between the topological and geometric filtration for smooth projective varieties. arXiv:math/0603203.

[L1] Lawson, H. B., Jr. Algebraic cycles and homotopy theory. Ann. of Math. (2) 129 (1989), no. 2, 253-291. MR986794 (90h:14008)

[Le] Lewis, J. D. A survey of the Hodge conjecture. Second edition. Appendix B by B. Brent Gordon. CRM Monograph Series, 10. American Mathematical Society, Providence, RI, 1999. MR 1683216 (2000a:14010)

[LF] Lima-Filho, P. Lawson homology for quasiprojective varieties. Compositio Math. 84 (1992), no. 1, 1-23. MR1183559 (93j:14007) 
[Ma] Manin, Ju. I. Correspondences, motifs and monoidal transformations (Russian). Mat. Sb. (N.S.) 77 (119), 1968, 475-507. MR0258836 (41:3482)

[Pe] Peters, C. Lawson homology for varieties with small Chow groups and the induced filtration on the Griffiths groups. Math. Z. 234 (2000), no. 2, 209-223. MR1765879 (2001f:14042)

[Ro] Roberts, Joel. Chow's moving lemma. Algebraic geometry, Oslo, 1970 (Proc. Fifth Nordic Summer School in Math.), pp. 89-96. Wolters-Noordhoff, Gröningen, 1972. MR0382269 (52:3154)

Department of Mathematics, Penn State Altoona, 3000 Ivyside Park, Altoona, PennSYLVANIA 16601

E-mail address: znie@psu.edu 